\section{It takes two to tango}

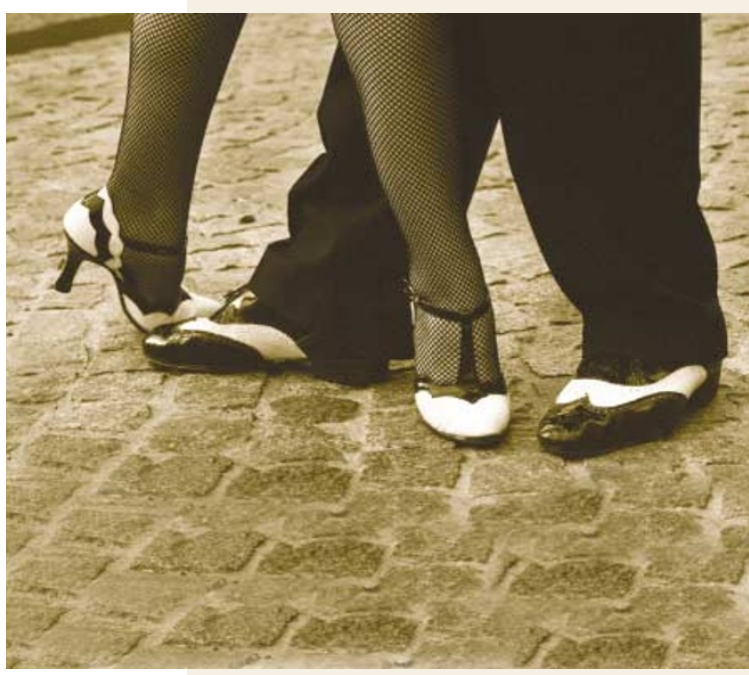

Much of the recent work on the development of active, specific immunotherapy of cancer has focused on the induction of $\mathrm{CD}^{+}$cytotoxic T-lymphocyte (CTL) responses, and several

\section{ONCOGENES}

\section{Fusion power}

Although many leukaemias have been associated with chromosomal translocations, the means by which a resulting fusion protein can transform a cell is not well understood. For instance, the AML1-ETO fusion protein, which is associated with acute myeloid leukaemia (AML), immortalizes haematopoietic progenitor cells by an unknown mechanism. In the July issue of Nature Medicine, Bryan Linggi et al. report that AML1-ETO can repress transcription of the ARF tumour suppressor.

The $t(8 ; 21)$ translocation, which is associated with $12-15 \%$ of all AML cases, fuses the DNA-binding domain of AML1 and the eighttwenty-one (ETO) corepressor. This fusion protein binds to the same DNA sequence as normal AML1, but functions as a transcriptional repressor instead of an activator. AML1-ETO expression extends the lifespan of primary myeloid progenitor cells in culture, so Linggi et al. investigated whether it affects components of the p53 checkpoint pathway.

The authors started by looking for AML1 binding sites in various gene promoters. studies have shown that it is possible to stimulate tumour-specific CTLs using dendritic cells (DCs) that are loaded with tumour antigens. But $\mathrm{CD}^{+} \mathrm{T}$-helper type 1 $\left(T_{H} 1\right)$ cells are also important components of effective immune responses. In this study, Schuler-Thurner and colleagues provide the first evidence that DCs that are loaded with tumour-specific peptides can rapidly induce $T_{H} 1$ responses in cancer patients that are readily detectable ex vivo.

The DCs that were used for vaccination in this study were derived from blood monocytes that were matured ex vivo using a defined cocktail (consisting of interleukin (IL)-1 $\beta$, IL-6, tumour-necrosis factor and prostaglandin $\mathrm{E}_{2}$ ) and cryopreserved before use. Aliquots of cells were thawed on the day of vaccination and loaded with various MHC class-I- and class-II-restricted peptides. Patients with incurable melanoma were treated with five biweekly vaccinations of DCs, followed by assessment one month after the final vaccination.
The results showed that the vaccination protocol induced a rapid $\mathrm{T}_{\mathrm{H}} 1$ response in patients, both to a control immunizing antigen and also to defined MHC class-IIrestricted tumour antigens. Immune responses were assessed on the basis of interferon- $\gamma$ production using ELISPOT assays and antigen-specific proliferative responses.

So, the results from this Phase I trial provide convincing evidence that cryopreserved DCs can induce $T_{H} 1$ responses against tumour antigens without significant toxicity, and it also encourages further development of DC-based vaccination technology.

$$
\begin{array}{r}
\text { Elaine Bell } \\
\text { Editor, Nature Reviews Immunology }
\end{array}
$$

\section{Q) References and links}

ORIGINAL RESEARCH PAPER Schuler-Thurner, B. et al. Rapid induction of tumor-specific type $1 \mathrm{~T}$ helper cells in metastatic melanoma patients by vaccination with mature, cryopreserved, peptide-loaded monocyte-derived dendritic cryopreserved, peptide-loaded monocyte-d
cells. J. Exp. Med. 195, 1279-1288 (2002)

cells. J. Exp. Med. 195, 1279-1288 (2002)
FURTHER READING Wolchok, J. D. \& Livingston, P. O. Vaccines for melanoma: translating basic immunology into new therapies. Lancet Oncol. 2, 205-211 (2001)

\section{WEB SITES}

Encyclopedia of Life Sciences: http://www.els.net dendritic cells (T-lymphocyte stimulating) Gerold Schuler's lab: http://www2.derma.med.unierlangen.de/klinik/mitarbeiter/schuler.htm
Although the TP53 gene (which encodes p53) itself does not have an AML1-binding site, the CDKN2A (which encodes ARF; also known as p14 in humans and p19 in mice) promoter contains eight AML1-binding sites. ARF antagonizes MDM2 to stabilize p53, and loss of ARF has been shown to impair p53-mediated growth arrest and apoptosis.

The authors found that expressing AML1 in cultured cells activates the transcription of the $\mathrm{ARF}$ transcript, and also induces senescence in fibroblasts. Expression of AML1-ETO, on the other hand, blocked AML1-mediated induction of the ARF-transcript promoter and repressed endogenous ARF-transcript expression, leading to immortalization. INK4a (also known as p16) has the same genetic locus as the ARF transcript, but is transcribed from a distinct promoter that lacks AML1-binding sites. Linggi et al. did not observe a significant difference in INK4A levels in cells expressing AML1-ETO, indicating that repression by this fusion protein is specific for ARF.

But is expression of the ARF transcript reduced in human leukaemia cells? Bonemarrow and blood samples from two cohorts of patients with $\mathrm{t}(8 ; 21)$-associated AML were independently tested for the expression of the ARF transcript by real-time polymerase chain reaction. These cells were found to express significantly less ARF-transcript mRNA than other types of leukaemia cells. The repression of ARF-transcript expression might therefore explain why TP53 is not mutated in these tumours. The authors suggest that ARF might also be repressed by other cancer-associated translocations that affect AML function.

Kristine Novak

(2) References and links

ORIGINAL RESEARCH PAPER Linggi, B. et al. The t(8;21) fusion protein, AML1-ETO, specifically represses the transcription of the $\mathrm{p} 14^{\mathrm{ARF}}$ tumor suppressor in acute myeloid leukemia. Nature Med. 7, 743-750 (2002)

FURTHER READING Licth, J. D. AML1 and the AML1-ETO FURTHER READING Licth, J. D. AML1 and the AML1-ETO
fusion protein in the pathogenesis of $\mathrm{t}(8 ; 21)$ AML. Oncogene 20, 5660-5679 (2001)

WEB SITE

Scott Hiebert's lab:

http://medschool.mc.vanderbilt.edu/hiebertlab/

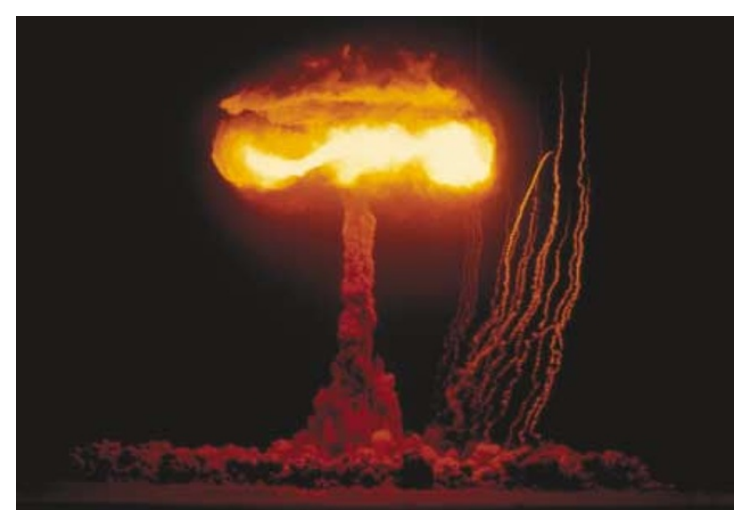

